\title{
CONTRIBUCIÓN AL ESTUDIO DE LAS ALGAS MARINAS BENTÓNICAS DE PUNTA ARENA Y CABO PULMO, BAJA CALIFORNIA SUR, MÉXICO ${ }^{1}$
}

\author{
Luz Elena Mateo-Cid \\ A. Catalina Mendoza-González ${ }^{2}$ \\ Citlalli Galicia García \\ Y \\ Laura Huerta Múzquiz \\ Departamento de Botánica \\ Escuela Nacional de Ciencias Biológicas, I.P.N. \\ Prolongación de Carpio y Plan de Ayala \\ 11340 México, D.F.
}

\begin{abstract}
RESUMEN
Se llevaron a cabo seis recolectas ocasionales en la zona submareal e intermareal de Punta Arena y Cabo Pulmo, Baja California Sur, entre 1989 y 1994. Se registró la presencia de 115 especies, 66 de ellas pertenecientes a la clase Rhodophyceae, 23 a Phaeophyceae, 4 a Bacillariophyceae, 18 a Chlorophyceae y 4 a Cyanophyceae. En Punta Arena se localizaron 88 especies y en Cabo Pulmo 68. La lista florística se acompaña de datos sobre estacionalidad, estadio reproductivo, nivel de marea, sustrato, exposición al oleaje y el epifitismo asociado. La ficoflora de estas localidades es de afinidad tropical, encontrándose más diversificada en primavera. Se citan por primera vez para el Golfo de California a Pneophyllum conicum (E. Y. Dawson) Keats, Chamberlain et Baba, Hincksia rallsiae (Vickers) P.C. Silva y Acetabularia parvula Solms-Laubach.
\end{abstract}

\section{ABSTRACT}

Benthic marine algae were collected at Punta Arena and Cabo Pulmo, Baja California Sur, México, between 1989 and 1994. The number of species found ascends to 115,4 belonging to Cyanophyceae, 66 to Rhodophyceae, 23 to Phaeophyceae, 4 to Bacillariophyceae and 18 to Chlorophyceae. 88 species were determined from Punta Arena and 68 from Cabo Pulmo. The floristic list includes data on reproductive stages, tidal levels and exposure to surf, substratum and epiphytism. The studied algal flora is of tropical affinity and has its greatest diversity in spring. Pneophyllum conicum (E. Y. Dawson) Keats, Chamberlain et Baba, Hincksia rallsiae (Vickers) P.C. Silva and Acetabularia parvula Solms-Laubach are new records for the Gulf of California.

\footnotetext{
${ }^{1}$ Trabajo parcialmente subsidiado por la Dirección de Estudios de Posgrado e Investigación del Instituto Politécnico Nacional. Clave 921793.

${ }^{2}$ Becario de la Comisión de Operación y Fomento de Actividades Académicas del I.P.N. (COFAA)
} 


\section{INTRODUCCIÓN}

Los primeros registros de algas marinas del Golfo de California fueron hechos por Hariot (1895); posteriormente Howe (1911) hizo una investigación más detallada. En 1924 Setchell y Gardner dieron a conocer un extenso inventario de plantas marinas del lugar y registraron más de 100 especies, obtenidas en la expedición que la Academia de Ciencias de California realizó en 1921. E. Y. Dawson llevó a cabo varios estudios sobre taxonomía y distribución de algas marinas en el Golfo y sus valiosos trabajos, publicados entre 1944 y 1966, enriquecieron el conocimiento sobre la flora marina de esa área.

En las contribuciones de Norris (1972), Norris y Norris (1973), Hollenberg y Norris (1977), Huerta-Múzquiz y Mendoza-González (1985), Mendoza-González y Mateo Cid (1986), Rocha-Ramírez y Siqueiros-Beltrones (1991) y Mateo-Cid et al. (1993) se encuentran numerosos registros de algas marinas de la porción septentrional del Golfo de California, de las costas de los estados de Sonora, Baja California y Baja California Sur. Respecto a la flora intermareal de la región sur del Golfo de California se han realizado menos estudios, existiendo aún lugares que no han sido explorados, como es el caso de Punta Arena. Por lo que se refiere a Cabo Pulmo, un total de 61 taxa han sido previamente citados, siendo los primeros registros los de Dawson (1949) quien menciona 13 especies; posteriormente esta región aparece referida en diferentes publicaciones de Dawson (1953a, 1954, 1958, 1962, 1963 a,b); Hollenberg (1961); Brusca y Thompson (1975); Norris y Johansen (1981) y recientemente Anaya y Riosmena (1996) añaden un inventario actualizado. Con el presente trabajo se busca ampliar el conocimiento florístico de la región sur del Golfo de California, proporcionando un primer listado comentado para Punta Arena, así como mayor información de las algas marinas bentónicas de Cabo Pulmo.

\section{ÁREA DE ESTUDIO}

Punta Arena se ubica en Bahía de la Ventana, en la costa sur-oriental de Baja California Sur, frente a la isla Cerralvo, a los $23^{\circ} 59^{\prime}$ de latitud N y $109^{\circ} 49^{\prime}$ de longitud $\mathrm{O}$ (Fig. 1). La zona de estudio tiene clima desértico, cálido y muy extremoso, del tipo BW(h') hw $\left(x^{\prime}\right)(e)$, la temperatura media anual es de $23.3^{\circ} \mathrm{C}$ y el promedio de la precipitación anual de $180.8 \mathrm{~mm}$ (García, 1981).

Cabo Pulmo se localiza en las coordenadas geográficas $23^{\circ} 26^{\prime} \mathrm{N}$ y $109^{\circ} 25^{\prime} \mathrm{O}$ y corresponde al ecosistema arrecifal más septentrional del litoral oriental del Océano Pacífico. Su ubicación en una zona marginal de desarrollo de este tipo de biocenosis y el hecho de ser el único arrecife coralino presente en el Golfo de California, determinan la relevancia de su estudio (Brusca y Thompson, 1975; Anaya y Riosmena, 1996).

El tipo de marea es mixto, con una desigualdad en las bajamares. Las mareas vivas ocurren en los meses de noviembre a febrero, del medio día al atardecer, la pleamar máxima es de + $0.580 \mathrm{~m}$ y la bajamar - $0.470 \mathrm{~m}$ (Anónimo, 1990); el litoral se encuentra influenciado por la corriente del Golfo de California y la Nor-ecuatorial. 
Mateo-Cid et al.: Contribución al Estudio de las Algas Marinas Bentónicas

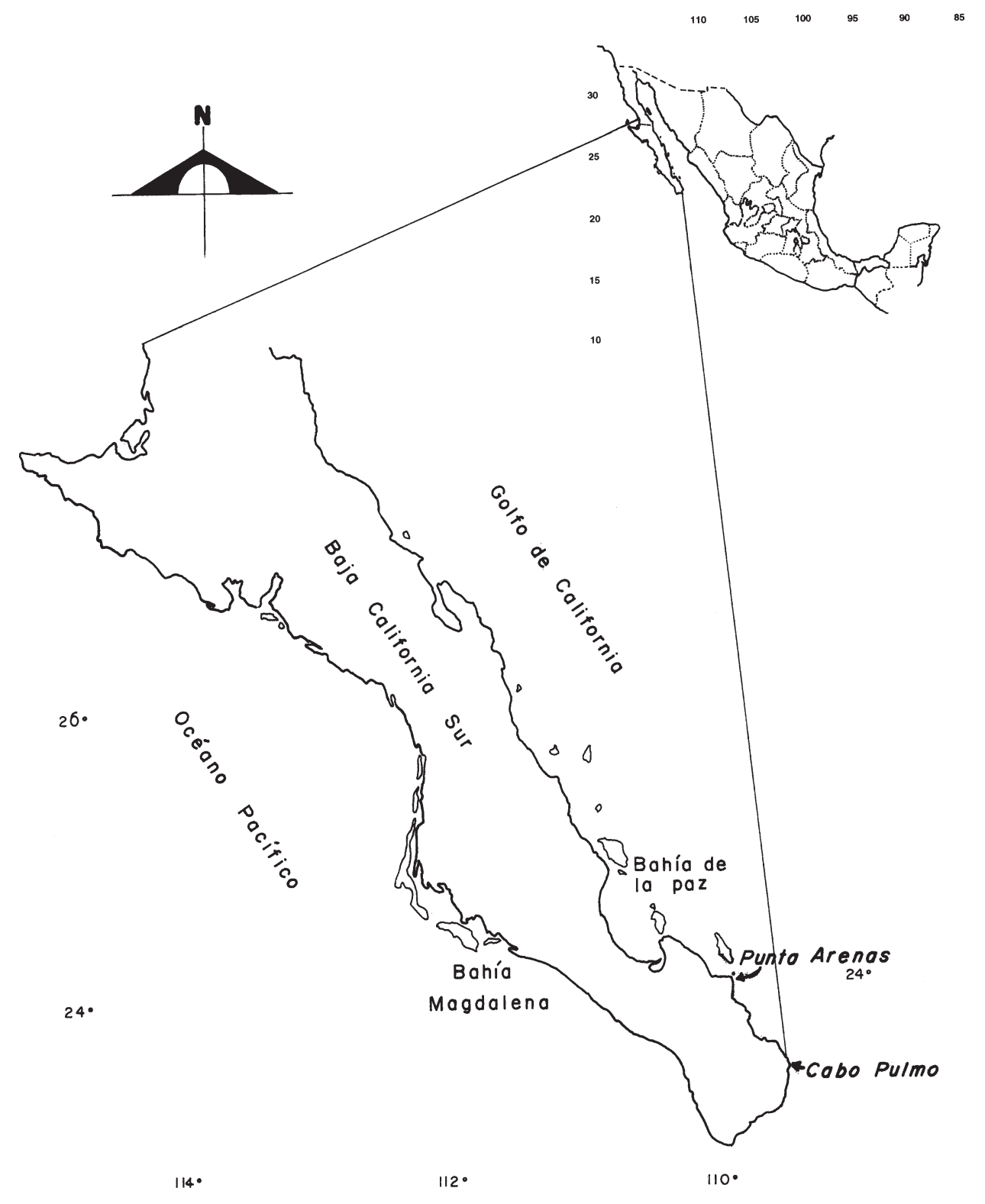

Fig. 1. Ubicación del área de estudio y de las localidades de muestreo. 
Localidades de muestreo

Punta Arena es una extensa playa arenosa con acantilados y agregados rocosos en su extremo norte. Los ejemplares se recolectaron en el nivel intermareal rocoso expuesto, en una extensión lineal aproximada de $600 \mathrm{~m}$, así como en pozas de marea de 0.5 a $2.0 \mathrm{~m}$ de ancho y 30-60 cm de profundidad. El fondo del lugar posee sustrato arenoso con pequeños agregados formados por restos de coral.

El arrecife de Cabo Pulmo posee varias barreras coralinas en cuyo fondo predomina la arena con fragmentos de coral antiguo y rocas. El material ficológico se obtuvo aproximadamente a 10 metros de la costa, en una pared arrecifal que termina en una zona rocosa expuesta situada al este del litoral; el tipo de sustrato que domina entre la costa y la barrera coralina es arenoso con restos de coral. El área de estudio abarca alrededor de $600 \mathrm{~m}$ de largo por $50 \mathrm{~m}$ de ancho.

\section{MÉTODOS}

Las muestras de algas se obtuvieron de los niveles intermareal y submareal en los meses de febrero, mayo, octubre de 1989 y abril de 1994 en Punta Arena; y en Cabo Pulmo en febrero de 1989, julio, diciembre de 1992 y abril de 1994.

Los especímenes se recolectaron a mano y por buceo libre entre 1 y $4 \mathrm{~m}$ de profundidad con ayuda de espátulas y navajas de campo; no se utilizó ningún método de muestreo en particular, pero se revisó exhaustivamente el área de estudio.

Las muestras ficológicas se fijaron en una solución de formalina a $5 \%$ en agua de mar y se trasladaron al Departamento de Botánica de la Escuela Nacional de Ciencias Biológicas del Instituto Politécnico Nacional. Para su determinación se utilizaron las obras de Setchell y Gardner (1924), Dawson (1953 a,b, 1954, 1959, 1960 a,b, 1961, 1962, 1963 a,b, 1966 a,c), Taylor (1945, 1960), Hollenberg (1961), Abbott y Hollenberg (1976), Norris y Johansen (1981), Anagnostidis y Kómarek (1988), Kómarek y Anagnostidis (1986), Pedroche y Silva (1996) y Kraft y Wynne (1996). Los ejemplares se encuentran depositados en la sección de Ficología del Herbario ENCB. La lista florística sigue el orden propuesto por Werner (1977), Wynne (1986), Anagnostidis y Kómarek (1988) y Silva et al. (1987). La actualización nomenclatural se basó en Cormaci y Furnari (1987), Kornman (1989), Koster (1955), Silva (1991), Silva et al. (1996) y Keats et al. (1997).

De cada especie registrada se incluyen datos relativos a su presencia en el área de estudio, estadio reproductivo, nivel de marea, ambiente marino, tipo de sustrato donde se localizó y los huéspedes en el caso de las especies epífitas (ver Cuadro 1). La clasificación del nivel de marea se basó en Dawson (1966a), Dawes (1986) y Lüning (1990).

\section{RESULTADOS Y DISCUSIÓN}

Florística

De las 115 especies de algas marinas determinadas, 66 (57.39\%) corresponden a Rhodophyceae, 23 (20\%) a Phaeophyceae, 4 (3.48\%) a Bacillariophyceae, 18 (15.65\%) a 
Chlorophyceae y 4 (3.48\%) a Cyanophyceae. La mayor riqueza se presentó en primavera (abril y mayo) con 84 especies, le siguió otoño (octubre) con 49, invierno (diciembre y febrero) con 48 y por último verano (julio) con 35 (ver Fig. 2).

Las familias Corallinaceae y Ceramiaceae de la clase Rhodophyceae fueron las mejor representadas en lo que se refiere a especies con 13 taxones cada una, de los cuales, cinco pertenecen a Amphiroa (Corallinaceae) y siete a Ceramium.

Con respecto a la clase Phaeophyceae, la familia Dictyotaceae contó con once especies, seis de ellas del género Dictyota. En Chlorophyceae, Caulerpaceae tiene tres especies de Caulerpa y los grupos con menor número de representantes fueron Cyanophyceae y Bacillariophyceae con cuatro cada uno. En Punta Arena se localizó un total de 88 especies (Cuadro 1); tres (2.6\%) de las cuales son nuevos registros para el Golfo de California, Pneophyllum conicum (E. Y. Dawson) Keats, Chamberlain et Baba, Hincksia rallsiae (Vickers) P.C. Silva y Acetabularia parvula Solms-Laubach (Cuadro 1, columna de observaciones como NCG).

Para Cabo Pulmo se inventariaron 68 especies, de las cuales 33 (48.5\%) constituyen nuevas citas para el arrecife, señaladas en la columna de observaciones del Cuadro 1. Los datos obtenidos en este estudio más los registros previos de Anaya y Riosmena (1996) indican que la flora ficológica conocida de Cabo Pulmo está conformada por 93 especies.

Al comparar el número de especies registradas en el presente estudio con las enlistadas para las Bahías de La Paz (Huerta-Múzquiz y Mendoza-González, 1985) y Concepción (Mateo-Cid et al., 1993), se observa que 57 de cada una de ellas se encuentran también en Punta Arena, lo que indica que 64\% de la flora de este último sitio tiene afinidad con las algas de las dos localidades de las partes central y sur del Golfo de California. Situación similar se observa en el caso de las especies de Cabo Pulmo, pues 39 de ellas han sido citadas de la Bahía de la Paz (Huerta-Múzquiz y Mendoza-González, 1985) y 40 de la Bahía Concepción (Mateo-Cid et al., 1993), alcanzando porcentajes de afinidad de $57.3 \%$ y de $58.8 \%$ respectivamente.

Como se observa en la Fig. 3, el mayor número de especies se presentó en Punta Arena (88), lo cual se debe a que este lugar se caracteriza por poseer litorales peñascosos, en los cuales se favorece la fijación de algas más diversas del ambiente marino. Es sabido, que las zonas rocosas son uno de los mejores hábitats para el crecimiento de las algas, debido a los factores ambientales que interactúan allí (Dawes, 1986). Por lo que respecta a los arrecifes coralinos de Cabo Pulmo, la flora marina es menos rica (68 especies) y se encuentra principalmente representada por algas calcificadas, como las pertenecientes a la familia Corallinaceae (Lüning, 1990).

Principales comunidades y su hábitat

El tipo de sustrato que predomina en Punta Arena, como ya se mencionó, es el rocoso. Como se observa en el Cuadro 1, las especies que se desarrollan preferentemente en estos hábitats son: Porphyra hollenbergii, Amphiroa beauvoisii, A. misakiensis, A. valonioides, Botryocladia uvarioides, Gracilaria pachydermatica, Dictyota crenulata, D. friabilis, Padina durvillaei, Cladophora sericea, Phyllodictyon anastomosans y Acetabularia parvula, entre otras. 


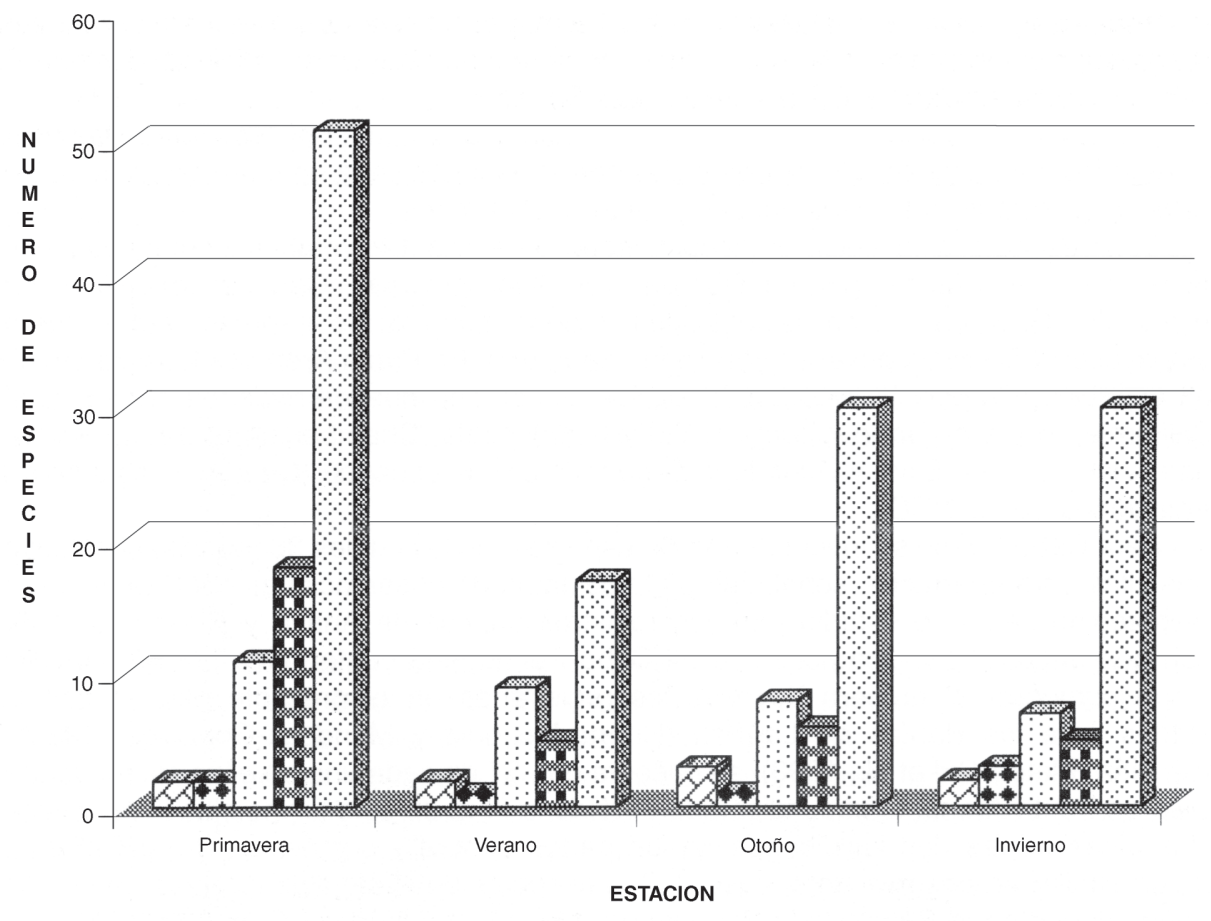

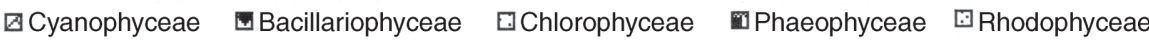

Fig. 2. Número de especies por clase en las diferentes estaciones del año.

En el nivel intermareal se localizaron depresiones o cavidades más o menos profundas que contienen agua de mar sujeta a renovación esporádica o constante, provocada por el oleaje y las mareas. Estas pozas de marea presentan condiciones diferentes a las de las rocas aledañas emergidas del mismo nivel, ya que en ellas los cambios de temperatura y salinidad son más drásticos a lo largo del día (Santelices, 1977).

En dicho microhábitat localizamos a Bangia atropurpurea, Gelidiella acerosa, Spongites decipiens, Gelidiopsis variabilis, Hypnea pannosa, Rhodymenia californica, Laurencia hancockii, Colpomenia sinuosa, Dictyota dichotoma, Ulva lactuca y Caulerpa sertularioides, entre otras.

En el caso de Cabo Pulmo, en la parte más expuesta del arrecife se desarrollan masas de rodofíceas incrustantes, limitando el establecimiento de las algas bentónicas; a su vez en las comunidades de madréporas, donde predomina la fauna, el hábitat resulta menos propicio para el establecimiento de esta flora (Díaz-Piferrer, 1967).

Round (1981) señala que en la clase Chlorophyceae las especies sifonadas son las que están mejor representadas en la zona interna de la planicie arrecifal, lugar conocido 
como intermareal, que es habitado por algas de este grupo que toleran factores ambientales rigurosos, tales como una fluctuación drástica de la temperatura y de la salinidad, desecación periódica, alta intensidad lumínica y exposición al viento y a la lluvia (Dawes, 1986).

En Cabo Pulmo se distingue una clara zonación: a partir de la línea de la costa hacia

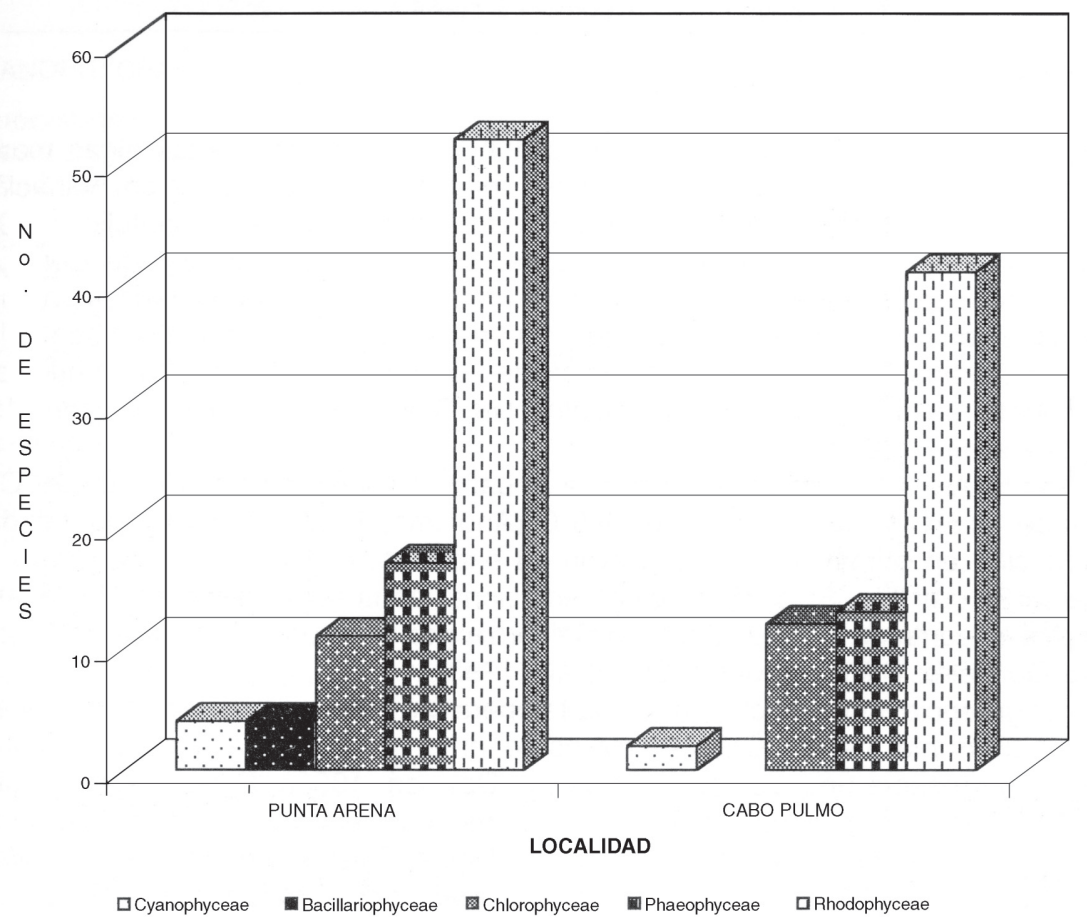

Fig. 3. Número de especies por clase y por localidad.

la barrera coralina se localiza una fitocenosis dominada por Cladophora prolifera, Padina mexicana y Ernodesmis verticillata, las que se desarrollan en rocas y fragmentos de coral; esta comunidad se ve afectada por los cambios en el nivel del agua provocados por el ritmo mareal (Lüning, 1990).

Después del nivel intermareal encontramos la plataforma arrecifal, la cual tiene una superficie irregular y una profundidad máxima de $1 \mathrm{~m}$; el sustrato está formado por fragmentos de coral y arena. En esta zona se registran numerosos representantes del orden Corallinales, principalmente de formas no articuladas como Spongites decipiens, Lithophyllum imitans, L. pallescens y Neogoniolithon trichotomum; además de Galaxaura marginata, Amphiroa vanbossea, A. beauvoisii, A. misakiensis y especies de Phaeophyta como Padina durvillaei y Dictyota crenulata. En la comunidad dominan las coralinas incrustantes, las cuales se localizaron durante todo el período de estudio; siendo las algas filamentosas y 
laminares las menos frecuentes. Esta situación coincide con lo encontrado por Littler et al. (1983) para el arrecife Carrie Bow en Belice, quienes observaron que los taxa calcáreos costrosos y articulados son más resistentes al pastoreo por peces, que las formas filamentosas y laminares.

Temperatura y luz

La temperatura y la luz determinan la distribución latitudinal de las algas marinas y por tanto su distribución geográfica; asimismo influyen en la composición, variación y periodicidad de las poblaciones en los niveles intermareal y submareal (Santelices, 1977).

Las costas de Baja California Sur, bañadas por las aguas del Mar de Cortés, se caracterizan por el exceso de evaporación sobre la precipitación. La vertiente de la península que da al Golfo tiene clima desértico caliente y un promedio de menos de 50 días de lluvia durante el año (Osorio-Taffall, 1943). Además existen diferencias estacionales pronunciadas en las temperaturas de las aguas superficiales del Golfo de California (Dawson, 1944, Pacheco-Ruiz et al., 1992).

En Punta Arena, la temperatura media anual superficial del agua es de $26^{\circ} \mathrm{C}$, en invierno es de $18.5^{\circ} \mathrm{C}$ y en el verano de $29.5^{\circ} \mathrm{C}$ (Anónimo, 1990). Como reflejo indudable de esta variación en primavera se localizaron 84 especies, mientras que en verano sólo se registraron 35 (ver Fig. 3). Esta sucesión estacional de la flora marina de Punta Arena es similar a la encontrada para el Golfo de California por Dawson (1944), Huerta-Múzquiz y Mendoza-González (1985) y Mateo-Cid et al. (1993).

En congruencia con el hecho de que la temperatura media anual superficial del agua en las costas de Punta Arena y Cabo Pulmo es del orden de $26{ }^{\circ} \mathrm{C}$, la ficoflora encontrada en este estudio presenta una distribución tropical (Dawson, 1966a; Dawes, 1986). Así, en la zona de estudio se hallaron representantes de los géneros Gelidium, Galaxaura, Grateloupia, Jania, Amphiroa, Laurencia, Dictyota, Padina, Sargassum, Halimeda, Caulerpa, Codium, Cladophora, Neomeris, Struveopsis, Acetabularia, Botryocladia, Gracilaria e Hypnea, de afinidad tropical (Lüning, 1990).

\section{Especies epífitas}

En este trabajo se encontraron 27 especies que crecen fijas sobre otras plantas, entre ellas: Stylonema alsidii, Anotrichium tenue, Ceramium camouii, C. sinicola, Ectocarpus gonodioides y Dictyopteris delicatula, (Cuadro 1).

De este conjunto de especies epífitas, 18 corresponden a Rhodophyceae, 3 a Phaeophyceae, 4 a Bacillariophyceae, 1 a Chlorophyceae y 1 a Cyanophyceae. El número más alto de ellas se encontró en primavera, cuando se registraron 11 algas distintas. 
Cuadro 1. Algas marinas bentónicas de Punta Arena y Cabo Pulmo, B.C.S.

\begin{tabular}{|c|c|c|c|c|c|c|c|c|}
\hline \multirow{2}{*}{ Especies } & \multicolumn{2}{|c|}{ Presencia } & \multirow{2}{*}{$\begin{array}{l}\text { Repro- } \\
\text { ducción }\end{array}$} & \multirow{2}{*}{$\begin{array}{l}\text { Nivel } \\
\text { de } \\
\text { marea }\end{array}$} & \multirow{2}{*}{$\begin{array}{l}\text { Exp. } \\
\text { al } \\
\text { oleaje }\end{array}$} & \multirow{2}{*}{$\begin{array}{l}\text { Sus- } \\
\text { trato }\end{array}$} & \multirow{2}{*}{$\begin{array}{c}\text { Epífita } \\
\text { de }\end{array}$} & \multirow[t]{2}{*}{ Obs. } \\
\hline & $\begin{array}{l}\text { Punta } \\
\text { Arena }\end{array}$ & $\begin{array}{l}\text { Cabo } \\
\text { Pulmo }\end{array}$ & & & & & & \\
\hline \multicolumn{9}{|l|}{ CYANOPHYCEAE } \\
\hline $\begin{array}{l}\text { Microcystaceae } \\
\text { 1. Microcystis zanardinii (Hauck) } \\
\text { P. C. Silva }\end{array}$ & $\mathrm{O}$ & & Ve & M & Ex & & 14 & $\mathrm{Ee}$ \\
\hline $\begin{array}{l}\text { Nostocaceae } \\
\text { 2. Anabaina pseudoscillatoria } \\
\text { Bory de Saint Vincent }\end{array}$ & $P$ & & $\mathrm{Ht}$ & M & D & $\mathrm{R}$ & & \\
\hline 3. Calothrix crustacea Thuret & 1 & VO & $\begin{array}{c}\mathrm{Ht} \mathrm{Ht} \\
\mathrm{Ht}\end{array}$ & L & Se & $\mathrm{R}$ & & $\stackrel{\mathrm{Pm}}{\mathrm{N}}$ \\
\hline $\begin{array}{l}\text { Oscillatoriaceae } \\
\text { 4. Blennothrix lyngbyacea (Küt- } \\
\text { zing) Anagnostidis et Komá- } \\
\text { rek }\end{array}$ & PVOI & PV & $\begin{array}{l}\mathrm{Hm} \mathrm{Hm} \\
\mathrm{Hm} \mathrm{Hm}\end{array}$ & L & Se & $\mathrm{R}$ & & $\mathrm{Pm}$ \\
\hline RHODOPHYCEAE & & & & & & & & \\
\hline $\begin{array}{l}\text { Porphyridiaceae } \\
\text { 5. Stylonema alsidii (Zanardini) } \\
\text { Drew }\end{array}$ & $P$ & & Ve & M & D & & 60 & $\mathrm{Ee}$ \\
\hline $\begin{array}{l}\text { Erythrotrichiaceae } \\
\text { 6. Sahlingia subintegra (Rosen- } \\
\text { vinge) Kornman }\end{array}$ & & V & Ve & L & Se & & 100 & $\begin{array}{l}\mathrm{Ee} \\
\mathrm{N}\end{array}$ \\
\hline $\begin{array}{l}\text { 7. Erythrotrichia carnea (Dillwyn) } \\
\text { J. Agardh }\end{array}$ & $P$ & & $\mathrm{Ve}$ & M & $\mathrm{Se}$ & & 102 & $\mathrm{Ee}$ \\
\hline $\begin{array}{l}\text { Bangiaceae } \\
\text { 8. Bangia atropurpurea (Roth) } \\
\text { C. Agardh }\end{array}$ & I & & $\mathrm{Mn}$ & L & $\mathrm{Se}$ & & & $\mathrm{Pm}$ \\
\hline $\begin{array}{l}\text { 9. Porphyra hollenbergii E. Y. } \\
\text { Dawson }\end{array}$ & 1 & & Gf & L & Ex & $\mathrm{R}$ & & \\
\hline $\begin{array}{l}\text { Acrochaetiaceae } \\
\text { 10. Acrochaetium microscopicum } \\
\text { (Nägeli ex Kützing) Nägeli }\end{array}$ & $P$ & & $\mathrm{Mn}$ & L & $\mathrm{Se}$ & & 102 & $\mathrm{Ee}$ \\
\hline $\begin{array}{l}\text { 11. A. savianum (Meneghini) } \\
\text { Nägeli }\end{array}$ & $P$ & & $\mathrm{Mn}$ & $M$ & D & & 93 & $\mathrm{Ee}$ \\
\hline $\begin{array}{l}\text { Galaxauraceae } \\
\text { 12. Galaxaura marginata (Ellis } \\
\text { et Solander) Lamouroux }\end{array}$ & & $P$ & Ve & L & Se & $\mathrm{R}$ & & \\
\hline $\begin{array}{l}\text { 13. G. oblongata (Ellis et Solan- } \\
\text { der) Lamouroux }\end{array}$ & $P$ & & $\mathrm{Ve}$ & $M$ & D & $\mathrm{R}$ & & \\
\hline
\end{tabular}


Cuadro 1. Continuación.

\begin{tabular}{|c|c|c|c|c|c|c|c|c|}
\hline \multirow[t]{2}{*}{ Especies } & \multicolumn{2}{|c|}{ Presencia } & \multirow{2}{*}{$\begin{array}{l}\text { Repro- } \\
\text { ducción }\end{array}$} & \multirow{2}{*}{$\begin{array}{l}\text { Nivel } \\
\text { de } \\
\text { marea }\end{array}$} & \multirow{2}{*}{$\begin{array}{l}\text { Exp. } \\
\text { al } \\
\text { oleaje }\end{array}$} & \multirow{2}{*}{$\begin{array}{l}\text { Sus- } \\
\text { trato }\end{array}$} & \multirow{2}{*}{$\begin{array}{l}\text { Epífita } \\
\text { de }\end{array}$} & \multirow[t]{2}{*}{ Obs. } \\
\hline & $\begin{array}{l}\text { Punta } \\
\text { Arena }\end{array}$ & $\begin{array}{l}\text { Cabo } \\
\text { Pulmo }\end{array}$ & & & & & & \\
\hline $\begin{array}{l}\text { Gelidiellaceae } \\
\text { 14. Gelidiella acerosa (Forsskäl) } \\
\text { Feldmann et Hamel }\end{array}$ & PVI & $\mathrm{O}$ & $\begin{array}{l}\text { Ve Te } \\
\text { Ve }\end{array}$ & $\begin{array}{l}\mathrm{L} \\
\mathrm{M}\end{array}$ & $\begin{array}{l}D \\
\text { Ex }\end{array}$ & $\mathrm{R}$ & & Pm \\
\hline $\begin{array}{l}\text { Pterocladiophilaceae } \\
\text { 15. Gelidiocolax microsphaerica } \\
\text { Gardner }\end{array}$ & $\mathrm{O}$ & & Ve & L & $\mathrm{Se}$ & 15 & & $\begin{array}{l}\text { PAR } \\
\mathrm{N}\end{array}$ \\
\hline $\begin{array}{l}\text { Gelidiaceae } \\
\text { 16. Gelidium johnstonii Setchell } \\
\text { et Gardner }\end{array}$ & $\mathrm{PO}$ & PVOI & $\begin{array}{l}\text { Te Te } \\
\text { Te Te }\end{array}$ & L & Ex & $\mathrm{R}$ & & \\
\hline $\begin{array}{l}\text { 17. G. pusillum (Stackhouse) } \\
\text { Le Jolis }\end{array}$ & I & $\mathrm{PO}$ & $\begin{array}{l}\mathrm{Te} \mathrm{Te} \\
\mathrm{Ve}\end{array}$ & L & $\mathrm{Se}$ & $\mathrm{R}$ & & $\mathrm{Pm}$ \\
\hline $\begin{array}{l}\text { 18. G. sclerophyllum W. R. } \\
\text { Taylor }\end{array}$ & I & & $\mathrm{Te}$ & L & $\mathrm{Se}$ & $\mathrm{R}$ & & $\mathrm{Pm}$ \\
\hline $\begin{array}{l}\text { Peyssonneliaceae } \\
\text { 19. Peyssonnelia orientalis } \\
\text { (Weber van Bosse) Cormar- } \\
\text { ci et Furnari }\end{array}$ & I & VO & $\begin{array}{l}\text { Ve Te } \\
\text { Ve }\end{array}$ & L & Ex & $\mathrm{R}$ & & $\mathrm{N}$ \\
\hline $\begin{array}{l}\text { Halymeniaceae } \\
\text { 20. Grateloupia versicolor (J. } \\
\text { Agardh) J. Agardh }\end{array}$ & POI & VO & $\begin{array}{l}\text { Ve Gf } \\
\text { Te }\end{array}$ & L & Se Ex & $\mathrm{R}$ & & $\mathrm{Pm}$ \\
\hline $\begin{array}{l}\text { 21. Prionitis abbreviata Setchell } \\
\text { et Gardner }\end{array}$ & & $\mathrm{O}$ & Ve & L & Ex & $\mathrm{R}$ & & $\mathrm{N}$ \\
\hline $\begin{array}{l}\text { Corallinaceae } \\
\text { 22. Amphiroa beauvoisii Lamou- } \\
\text { roux }\end{array}$ & PVOI & PVOI & $\begin{array}{l}\text { Bp Te } \\
\text { Te Bp }\end{array}$ & M & D & $\mathrm{R}$ & & \\
\hline 23. A. dimorpha Lemoine & $P$ & & $\mathrm{Te}$ & M & D & $\mathrm{R}$ & & \\
\hline 24. A. misakiensis Yendo & Ol & $\mathrm{Pv}$ & $\mathrm{Te} \mathrm{Te}$ & M & $\mathrm{Se}$ & $\mathrm{R}$ & & $\mathrm{Pm}$ \\
\hline 25. A. valonioides Yendo & $\mathrm{Pl}$ & $\mathrm{PO}$ & $\begin{array}{l}\text { Ve Te } \\
\text { Bp }\end{array}$ & M & $\mathrm{D} \mathrm{Se}$ & $\mathrm{R}$ & & $\mathrm{N}$ \\
\hline 26. A. vanbossea Lemoine & & $\mathrm{PO}$ & $\mathrm{Te} \mathrm{Ve}$ & M & $\mathrm{Se}$ & $\mathrm{R}$ & & $\mathrm{N}$ \\
\hline 27. Jania adhaerens Lamouroux & I & $\mathrm{PO}$ & $\begin{array}{l}\text { Te Ve } \\
\mathrm{Ve}\end{array}$ & $\mathrm{L}$ & $\mathrm{Se}$ & $\mathrm{R}$ & & $\mathrm{Pm}$ \\
\hline 28. Lithophyllum imitans Foslie & PVOI & $\mathrm{PO}$ & $\begin{array}{l}\text { Te Te } \\
\text { Te Te }\end{array}$ & M & D & $\mathrm{R}$ & & $\mathrm{Pm}$ \\
\hline
\end{tabular}


Cuadro 1. Continuación.

\begin{tabular}{|c|c|c|c|c|c|c|c|c|}
\hline \multirow{2}{*}{ Especies } & \multicolumn{2}{|c|}{ Presencia } & \multirow{2}{*}{$\begin{array}{l}\text { Repro- } \\
\text { ducción }\end{array}$} & \multirow{2}{*}{$\begin{array}{c}\text { Nivel } \\
\text { de } \\
\text { marea }\end{array}$} & \multirow{2}{*}{$\begin{array}{l}\text { Exp. } \\
\text { al } \\
\text { oleaje }\end{array}$} & \multirow{2}{*}{$\begin{array}{l}\text { Sus- } \\
\text { trato }\end{array}$} & \multirow{2}{*}{$\begin{array}{c}\text { Epífita } \\
\text { de }\end{array}$} & \multirow{2}{*}{ Obs. } \\
\hline & $\begin{array}{l}\text { Punta } \\
\text { Arena }\end{array}$ & $\begin{array}{l}\text { Cabo } \\
\text { Pulmo }\end{array}$ & & & & & & \\
\hline 29. L. pallescens (Foslie) Foslie & & PVOI & $\begin{array}{l}\text { Te Te } \\
\text { Te Te }\end{array}$ & M & D & $\mathrm{R}$ & & $\mathrm{N}$ \\
\hline $\begin{array}{l}\text { 30. Neogoniolithon setchellii } \\
\text { (Foslie) Adey }\end{array}$ & $\mathrm{POI}$ & Vo & $\begin{array}{l}\mathrm{Te} \mathrm{Te} \\
\mathrm{Ve}\end{array}$ & $\mathrm{L}$ & Se & $\mathrm{R}$ & & $\mathrm{Pm}$ \\
\hline $\begin{array}{l}\text { 31. N. trichotomum (Heydrich) } \\
\text { Setchell et Mason }\end{array}$ & $\mathrm{PO}$ & PVOI & $\begin{array}{l}\text { Te Te } \\
\text { Te Te }\end{array}$ & M & D & $\mathrm{R}$ & & N \\
\hline $\begin{array}{l}\text { 32. Pneophyllum conicum (E. Y. } \\
\text { Dawson) Keats, Chamberlain } \\
\text { et Baba }\end{array}$ & PV & $\mathrm{P}$ & $\mathrm{Te} \mathrm{Te}$ & $\begin{array}{l}M \\
L\end{array}$ & D & $\mathrm{R}$ & & $\begin{array}{l}\text { Pm } \\
\text { NCG }\end{array}$ \\
\hline 33. Pneophyllum fragile Kützing & $\mathrm{P}$ & $\mathrm{PO}$ & $\begin{array}{c}\text { Te } \mathrm{Gm} \\
\mathrm{Gf}\end{array}$ & M & Ex & & 3541 & $\mathrm{~N}$ \\
\hline $\begin{array}{l}\text { 34. Spongites decipiens (Foslie) } \\
\text { Chamberlain }\end{array}$ & PVI & $\mathrm{P}$ & Te Bp & $\begin{array}{l}M \\
L\end{array}$ & D & $\mathrm{R}$ & & Pm \\
\hline $\begin{array}{l}\text { Gracilariaceae } \\
\text { 35. Gracilaria pachydermatica } \\
\text { Setchell et Gardner }\end{array}$ & POI & $\mathrm{O}$ & $\begin{array}{l}\text { Te Gf } \\
\text { Ve }\end{array}$ & $\mathrm{L}$ & Ex & $\mathrm{R}$ & & $\stackrel{\mathrm{Pm}}{\mathrm{N}}$ \\
\hline 36. G. turgida E. Y. Dawson & & $P$ & Gf & M & Se & $\mathrm{R}$ & & $\mathrm{N}$ \\
\hline 37. G. veleroae E. Y. Dawson & $\mathrm{PI}$ & & Ve & M & $\mathrm{D}$ & $\mathrm{R}$ & & $\mathrm{Pm}$ \\
\hline $\begin{array}{l}\text { Hypneaceae } \\
\text { 38. Hypnea johnstonii Setchell } \\
\text { et Gardner }\end{array}$ & $P$ & & $\mathrm{Te}$ & L & Ex & $\mathrm{R}$ & & \\
\hline 39. H. pannosa J. Agardh & $\mathrm{POI}$ & $\mathrm{PO}$ & $\mathrm{Ve}$ & L & $\mathrm{Se}$ & $\mathrm{R}$ & & $\mathrm{Pm}$ \\
\hline $\begin{array}{l}\text { 40. H. spinella (C. Agardh) Küt- } \\
\text { zing }\end{array}$ & PI & 0 & $\mathrm{Ve}$ & $\begin{array}{l}\mathrm{L} \\
\mathrm{M}\end{array}$ & $\begin{array}{l}\text { Se } \\
\text { D }\end{array}$ & $\mathrm{R}$ & & $\mathrm{Pm}$ \\
\hline $\begin{array}{l}\text { Phyllophoraceae } \\
\text { 41. Gymnogongrus johnstonii } \\
\text { (Setchell et Gardner) E. Y. } \\
\text { Dawson }\end{array}$ & PVO & $\mathrm{POI}$ & $\begin{array}{c}\text { Ci Gf } \mathrm{Ci} \\
\mathrm{Ve}\end{array}$ & L & Ex & $\mathrm{R}$ & & $\mathrm{N}$ \\
\hline $\begin{array}{l}\text { Gigartinaceae } \\
\text { 42. Chondracanthus intermedius } \\
\text { (Suringar) Hommersand }\end{array}$ & & PO & $\mathrm{Ve} T e$ & $M$ & Ex & $\mathrm{R}$ & & $\mathrm{N}$ \\
\hline $\begin{array}{l}\text { Rhodymeniaceae } \\
\text { 43. Botryocladia uvarioides E. Y. } \\
\text { Dawson }\end{array}$ & $P$ & & $\mathrm{Ve}$ & L & Ex & $\mathrm{R}$ & & \\
\hline $\begin{array}{l}\text { 44. Gelidiopsis variabilis } \\
\text { (J. Agardh) F. Schmitz }\end{array}$ & Ol & 0 & $\mathrm{Te} \mathrm{Ve}$ & L & $\begin{array}{l}\text { Ex } \\
\text { Se }\end{array}$ & $\mathrm{R}$ & & $\mathrm{Pm}$ \\
\hline
\end{tabular}


Cuadro 1. Continuación.

\begin{tabular}{|c|c|c|c|c|c|c|c|c|}
\hline \multirow[t]{2}{*}{ Especies } & \multicolumn{2}{|c|}{ Presencia } & \multirow{2}{*}{$\begin{array}{l}\text { Repro- } \\
\text { ducción }\end{array}$} & \multirow{2}{*}{$\begin{array}{l}\text { Nivel } \\
\text { de } \\
\text { marea }\end{array}$} & \multirow{2}{*}{$\begin{array}{l}\text { Exp. } \\
\text { al } \\
\text { oleaje }\end{array}$} & \multirow{2}{*}{$\begin{array}{l}\text { Sus- } \\
\text { trato }\end{array}$} & \multirow{2}{*}{$\begin{array}{l}\text { Epífita } \\
\text { de }\end{array}$} & \multirow[t]{2}{*}{ Obs. } \\
\hline & $\begin{array}{l}\text { Punta } \\
\text { Arena }\end{array}$ & $\begin{array}{l}\text { Cabo } \\
\text { Pulmo }\end{array}$ & & & & & & \\
\hline 45. Rhodymenia californica Kylin & I & & Ve & L & $\mathrm{Se}$ & $\mathrm{R}$ & & $\mathrm{Pm}$ \\
\hline $\begin{array}{l}\text { Lomentariaceae } \\
\text { 46. Lomentaria hakodatensis } \\
\text { Yendo? }\end{array}$ & & $\mathrm{P}$ & Ve & M & $\mathrm{D}$ & & 24 & $\begin{array}{c}\mathrm{N} \\
\mathrm{EE}\end{array}$ \\
\hline Champiaceae & & & & & & & & \\
\hline $\begin{array}{l}\text { 47. Champia parvula (C. Agardh) } \\
\text { Harvey }\end{array}$ & $P$ & $\mathrm{PO}$ & Ve Te & L M & Ex Se & $\mathrm{R}$ & 54 & \\
\hline $\begin{array}{l}\text { Ceramiaceae } \\
\text { 48. Anotrichium tenue (C. } \\
\text { Agardh) Nägeli }\end{array}$ & I & $\mathrm{P}$ & Te Ve & M L & $\mathrm{Se}$ & & $\begin{array}{l}13 \\
27\end{array}$ & \\
\hline $\begin{array}{l}\text { 49. Antithamnionella elegans } \\
\text { (Berthold) Prince et John }\end{array}$ & & $\mathrm{PO}$ & Te Ve & M L & $\mathrm{Se}$ & & 35 & $\mathrm{Ee}$ \\
\hline $\begin{array}{l}\text { 50. Centroceras clavulatum } \\
\text { (C. Agardh) Montagne }\end{array}$ & PV & $\mathrm{P}$ & Te Ve & M L & $\begin{array}{l}\mathrm{D} \\
\mathrm{Se}\end{array}$ & $\mathrm{R}$ & $\begin{array}{l}47 \\
37\end{array}$ & \\
\hline $\begin{array}{l}\text { 51. Ceramium comouii E. Y. } \\
\text { Dawson }\end{array}$ & $P$ & & Te, Gf & M & $\mathrm{D}$ & & 36 & $\mathrm{Ee}$ \\
\hline 52. C. equisetoides E. Y. Dawson & I & & $\mathrm{Te}$ & L & $\mathrm{Se}$ & & $\begin{array}{l}17 \\
\mathrm{Ee}\end{array}$ & Pm \\
\hline $\begin{array}{l}\text { 53. C. flaccidum (Kützing) } \\
\text { Ardissone }\end{array}$ & $P$ & $\mathrm{P}$ & $\mathrm{Te}$ & M L & Se D & $\mathrm{R}$ & 13 & $\mathrm{Pm}$ \\
\hline 54. C. monacanthum J. Agardh & $\mathrm{PO}$ & & Te Ve & M & D & & 39 & $\mathrm{Ee}$ \\
\hline $\begin{array}{l}\text { 55. C. procumbens Setchell et } \\
\text { Gardner }\end{array}$ & & $\mathrm{P}$ & $\mathrm{Te}$ & $M$ & D & & 84 & Ee N \\
\hline $\begin{array}{l}\text { 56. C. sinicola Setchell et } \\
\text { Gardner }\end{array}$ & $P$ & & $\mathrm{Te}$ & L & $\mathrm{Se}$ & & 15 & $\mathrm{Ee}$ \\
\hline $\begin{array}{l}\text { 57. C. zacae Setchell et } \\
\text { Gardner }\end{array}$ & I & & & L & $\mathrm{Se}$ & $\mathrm{R}$ & & \\
\hline $\begin{array}{l}\text { 58. Lejolisea columbiana W. R. } \\
\text { Taylor }\end{array}$ & & V & & M & D & $\mathrm{R}$ & & \\
\hline $\begin{array}{l}\text { 59. Pleonosporium globuliferum } \\
\text { Levring }\end{array}$ & $\mathrm{P}$ & & Ps & L & $\mathrm{Se}$ & $\mathrm{R}$ & 15 & $\mathrm{Ee}$ \\
\hline $\begin{array}{l}\text { 60. Spyridia filamentosa (Wulfen) } \\
\text { Harvey }\end{array}$ & $P$ & O & $\mathrm{Te} \mathrm{Ve}$ & $M$ & Se D & $\mathrm{R}$ & & \\
\hline
\end{tabular}


Cuadro 1. Continuación.

\begin{tabular}{|c|c|c|c|c|c|c|c|c|}
\hline \multirow[t]{2}{*}{ Especies } & \multicolumn{2}{|c|}{ Presencia } & \multirow{2}{*}{\begin{tabular}{|l|} 
Repro- \\
ducción
\end{tabular}} & \multirow{2}{*}{$\begin{array}{c}\text { Nivel } \\
\text { de } \\
\text { marea }\end{array}$} & \multirow{2}{*}{$\begin{array}{l}\text { Exp. } \\
\text { al } \\
\text { oleaje }\end{array}$} & \multirow{2}{*}{$\begin{array}{l}\text { Sus- } \\
\text { trato }\end{array}$} & \multirow{2}{*}{$\begin{array}{c}\text { Epífita } \\
\text { de }\end{array}$} & \multirow{2}{*}{ Obs. } \\
\hline & $\begin{array}{l}\text { Punta } \\
\text { Arena }\end{array}$ & $\begin{array}{l}\text { Cabo } \\
\text { Pulmo }\end{array}$ & & & & & & \\
\hline $\begin{array}{l}\text { Dasyaceae } \\
\text { 61. Dasya sinicola (Setchell et } \\
\text { Gardner) E. Y. Dawson var. } \\
\text { sinicola }\end{array}$ & $\mathrm{P}$ & $\mathrm{PO}$ & $\mathrm{Ve} \mathrm{Te}$ & L & Ex & $\mathrm{R}$ & & \\
\hline $\begin{array}{l}\text { 62. Heterosiphonia crispella var. } \\
\text { laxa (Boergesen) Wynne }\end{array}$ & $\mathrm{PI}$ & & $\mathrm{Ve} \mathrm{Te}$ & M L & $\begin{array}{l}\text { Ex } \\
D\end{array}$ & $\mathrm{R}$ & & $\mathrm{Pm}$ \\
\hline $\begin{array}{l}\text { Rhodomelaceae } \\
\text { 63. Chondria acrorhizophora } \\
\text { Setchell et Gardner }\end{array}$ & $P$ & & $\mathrm{Te}$ & L & Ex & $\mathrm{R}$ & & \\
\hline $\begin{array}{l}\text { 64. Digenia simplex (Wulfen) C. } \\
\text { Agardh }\end{array}$ & & $P$ & $\mathrm{Ve}$ & M & D & $\mathrm{R}$ & & $\mathrm{N}$ \\
\hline $\begin{array}{l}\text { 65. Herposiphonia hollenbergii } \\
\text { E. Y. Dawson }\end{array}$ & & VI & $\mathrm{Ve} V \mathrm{~V}$ & M & $\mathrm{D}$ & $\mathrm{R}$ & & $\mathrm{N}$ \\
\hline $\begin{array}{l}\text { 66. Laurencia hancockii E. Y. } \\
\text { Dawson }\end{array}$ & I & & $\mathrm{Te}$ & L & Se & & 36 & $\begin{array}{l}\mathrm{Ee} \\
\mathrm{Pm}\end{array}$ \\
\hline 67. L. Iajolla E. Y. Dawson & $\mathrm{P}$ & O & $\mathrm{Te} \mathrm{Te}$ & M & Se $D$ & $\mathrm{R}$ & & $\mathrm{N}$ \\
\hline 68. L. voragina W. R. Taylor & $\mathrm{O}$ & & $\mathrm{Te}$ & M & Se & $\mathrm{R}$ & & $\mathrm{N}$ \\
\hline $\begin{array}{l}\text { 69. Polysiphonia scopulorum } \\
\text { var. villum (J. Agardh) } \\
\text { Hollenberg }\end{array}$ & $P$ & & $\mathrm{Te}$ & M & D & $\mathrm{R}$ & & \\
\hline 70. P. simplex Hollenberg & & $P$ & $\mathrm{Te}$ & M & Se & $\mathrm{R}$ & & \\
\hline PHAEOPHYCEAE & & & & & & & & \\
\hline $\begin{array}{l}\text { Ectocarpaceae } \\
\text { 71. Ectocarpus chantrasoides } \\
\text { Setchell et Gardner }\end{array}$ & & $P$ & $\mathrm{Pp}$ & M & $\mathrm{D}$ & & 61 & Ee $N$ \\
\hline $\begin{array}{l}\text { 72. E. gonodioides Setchell et } \\
\text { Gardner }\end{array}$ & 1 & & $\mathrm{Pp}$ & M & $\mathrm{Se}$ & & 69 & $\mathrm{Ee}$ \\
\hline $\begin{array}{l}\text { 73. E. simulans Setchell et } \\
\text { Gardner }\end{array}$ & & V & $\mathrm{Pp}$ & M & se & $\mathrm{R}$ & & $\mathrm{N}$ \\
\hline $\begin{array}{l}\text { 74. Hincksia rallsiae (Vickers) } \\
\text { P. C. Silva }\end{array}$ & $P$ & & $\mathrm{Pp}$ & M & $\mathrm{D}$ & $\mathrm{R}$ & & NCG \\
\hline $\begin{array}{l}\text { Ralfsiaceae } \\
\text { 75. Ralfsia confusa Hollenberg }\end{array}$ & & $P$ & Uu & L & Se & $\mathrm{R}$ & & $\mathrm{N}$ \\
\hline 76. R. hancockii E. Y. Dawson & 1 & & Uu & L & $\mathrm{Se}$ & $\mathrm{R}$ & & $\mathrm{Pm}$ \\
\hline
\end{tabular}


Cuadro 1. Continuación.

\begin{tabular}{|c|c|c|c|c|c|c|c|c|}
\hline \multirow{2}{*}{ Especies } & \multicolumn{2}{|c|}{ Presencia } & \multirow{2}{*}{$\begin{array}{l}\text { Repro- } \\
\text { ducción }\end{array}$} & \multirow{2}{*}{$\begin{array}{l}\text { Nivel } \\
\text { de } \\
\text { marea }\end{array}$} & \multirow{2}{*}{$\begin{array}{l}\text { Exp. } \\
\text { al } \\
\text { oleaje }\end{array}$} & \multirow{2}{*}{$\begin{array}{l}\text { Sus- } \\
\text { trato }\end{array}$} & \multirow{2}{*}{$\begin{array}{c}\text { Epífita } \\
\text { de }\end{array}$} & \multirow[t]{2}{*}{ Obs. } \\
\hline & $\begin{array}{l}\text { Punta } \\
\text { Arena }\end{array}$ & $\begin{array}{l}\text { Cabo } \\
\text { Pulmo }\end{array}$ & & & & & & \\
\hline 77. R. pacifica Hollenberg & & $\mathrm{O}$ & $\mathrm{Uu}$ & $\mathrm{L}$ & $\mathrm{Se}$ & $\mathrm{R}$ & & $\mathrm{N}$ \\
\hline $\begin{array}{l}\text { Cutleriaceae } \\
\text { 78. Cutleria hancockii E. Y. } \\
\text { Dawson }\end{array}$ & $P$ & & Gf $\mathrm{Gm}$ & L & Ex & $\mathrm{R}$ & & $\mathrm{Pm}$ \\
\hline $\begin{array}{l}\text { Scytosiphoniaceae } \\
\text { 79. Colpomenia sinuosa (Roth) } \\
\text { Derbés et Solier }\end{array}$ & $P$ & & Ve & L & Ex & $\mathrm{R}$ & & $\mathrm{Pm}$ \\
\hline 80. C. tuberculata Saunders & $P$ & $P$ & Ve & M L & $\mathrm{D} \mathrm{Se}$ & $\mathrm{R}$ & & $\mathrm{N}$ \\
\hline $\begin{array}{l}\text { 81. Rosenvingea intricata (J. } \\
\text { Agardh) Boergesen }\end{array}$ & $\mathrm{P}$ & & Ve & M & $\mathrm{D}$ & $\mathrm{R}$ & & \\
\hline $\begin{array}{l}\text { Dictyotaceae } \\
\text { 82. Dictyopteris delicatula } \\
\text { Lamouroux }\end{array}$ & $P$ & & Ve & M & D & & 27 & $\mathrm{Ee}$ \\
\hline 83. Dictyota cervicornis Kützing & $\mathrm{P}$ & & Gf & M & D Ex & $\mathrm{R}$ & & \\
\hline 84. D. crenulata J. Agardh & POV & $\mathrm{PO}$ & $\begin{array}{l}\text { Ve Gf } \\
\text { Ve }\end{array}$ & $M L$ & D & $\mathrm{R}$ & & \\
\hline $\begin{array}{l}\text { 85. D. dichotoma (Hudson) } \\
\text { Lamouroux }\end{array}$ & $\mathrm{PI}$ & $\mathrm{v}$ & Gf & M L & D & $\mathrm{R}$ & & $\mathrm{Pm}$ \\
\hline 86. D. divaricata Lamouroux & $\mathrm{PI}$ & $\mathrm{O}$ & Gf & L M & D & $\mathrm{R}$ & & \\
\hline 87. D. friabilis Setchell & $P$ & 0 & Ve Gf & M L & D Ex & $\mathrm{R}$ & & \\
\hline $\begin{array}{l}\text { 88. D. pinnata (E. Y. Dawson) } \\
\text { Horning, Schneiter et Prud' } \\
\text { Homme van Reine }\end{array}$ & $P$ & & Ve & $M$ & D & $\mathrm{R}$ & & \\
\hline $\begin{array}{l}\text { 89. Lobophora variegata (Lamou } \\
\text { roux) Womersley ex P. C. } \\
\text { Silva, Meñez et Moe }\end{array}$ & & $P$ & Ve & $M$ & $\mathrm{Se}$ & $\mathrm{R}$ & & $\mathrm{N}$ \\
\hline $\begin{array}{l}\text { 90. Padina gymnospora (Küt- } \\
\text { zing) Sonder }\end{array}$ & $P$ & & Gf & $M$ & D & $\mathrm{R}$ & & \\
\hline $\begin{array}{l}\text { 91. P. durvillaei Bory de Saint- } \\
\text { Vincent }\end{array}$ & PV & PV & Gf Gf & $M$ & $D$ & $\mathrm{R}$ & & \\
\hline 92. P. mexicana E. Y. Dawson & & $\mathrm{O}$ & Gf & M & D & $\mathrm{R}$ & & \\
\hline $\begin{array}{l}\text { Sargassaceae } \\
\text { 93. Sargassum sinicola Setchell } \\
\text { et Gardner }\end{array}$ & PV & $\mathrm{POI}$ & $\begin{array}{l}\text { Ve Gf } \\
\text { Ve }\end{array}$ & M L & D Ex & $\mathrm{R}$ & & \\
\hline
\end{tabular}


Cuadro 1. Continuación.

\begin{tabular}{|c|c|c|c|c|c|c|c|c|}
\hline \multirow[t]{2}{*}{ Especies } & \multicolumn{2}{|c|}{ Presencia } & \multirow{2}{*}{$\begin{array}{l}\text { Repro- } \\
\text { ducción }\end{array}$} & \multirow{2}{*}{$\begin{array}{c}\text { Nivel } \\
\text { de } \\
\text { marea }\end{array}$} & \multirow{2}{*}{$\begin{array}{l}\text { Exp. } \\
\text { al } \\
\text { oleaje }\end{array}$} & \multirow{2}{*}{$\begin{array}{l}\text { Sus- } \\
\text { trato }\end{array}$} & \multirow{2}{*}{$\begin{array}{c}\text { Epífita } \\
\text { de }\end{array}$} & \multirow[t]{2}{*}{ Obs. } \\
\hline & $\begin{array}{l}\text { Punta } \\
\text { Arena }\end{array}$ & $\begin{array}{l}\text { Cabo } \\
\text { Pulmo }\end{array}$ & & & & & & \\
\hline \multicolumn{9}{|l|}{ BACILLARIOPHYCEAE } \\
\hline $\begin{array}{l}\text { Discaceae } \\
\text { 94. Melosira juergensii C. } \\
\text { Agardh }\end{array}$ & $P$ & & Ve & L & Se & & 90 & $\mathrm{Ee}$ \\
\hline $\begin{array}{l}\text { Fragilariaceae } \\
\text { 95. Grammatophora marina } \\
\text { (Lyngbye) Kützing }\end{array}$ & PVI & & Ve & L & Se & & $\begin{array}{ll}41 & 53 \\
60 & 80\end{array}$ & \\
\hline $\begin{array}{l}\text { 96. Licmophora flabellata } \\
\text { (Carmichael) C. Agardh }\end{array}$ & $\mathrm{OI}$ & & $\mathrm{Ve}$ & L & $\mathrm{Se}$ & & 39 & $\begin{array}{c}\mathrm{Pm} \\
\mathrm{Ee}\end{array}$ \\
\hline $\begin{array}{l}\text { Achnantaceae } \\
\text { 97. Cocconeis placentula } \\
\text { Ehrenberg }\end{array}$ & 1 & & Ve & L & Se & & 341 & $\mathrm{Pm}$ \\
\hline CHLOROPHYCEAE & & & & & & & & \\
\hline $\begin{array}{l}\text { Ulvaceae } \\
\text { 98. Enteromorpha clathrata } \\
\text { (Roth) Greville }\end{array}$ & 1 & & Ve & L & Se & $\mathrm{R}$ & & $\mathrm{Pm}$ \\
\hline 99. Ulva lactuca Linnaeus & $\mathrm{Pi}$ & PV & $\begin{array}{l}\mathrm{Ve} V \mathrm{Ve} \\
\mathrm{Ve}\end{array}$ & L M & Se Ex & $\mathrm{R}$ & & $\mathrm{Pm}$ \\
\hline $\begin{array}{l}\text { Cladophoraceae } \\
\text { 100. Chaetomorpha antennina } \\
\text { (Bory de Saint Vincent) } \\
\text { Kützing }\end{array}$ & & V & Ve & M & Ex & $\mathrm{R}$ & & $\mathrm{N}$ \\
\hline $\begin{array}{l}\text { 101. Cladophora prolifera (Roth) } \\
\text { Kützing }\end{array}$ & & $\mathrm{O}$ & Ve & M & Ex & $\mathrm{R}$ & & N \\
\hline $\begin{array}{l}\text { 102. C. sericea (Hudson) Küt- } \\
\text { zing }\end{array}$ & $P$ & V & $\mathrm{Ve} V \mathrm{Ve}$ & M L & $\mathrm{D} \mathrm{Se}$ & $\mathrm{R}$ & & N \\
\hline $\begin{array}{l}\text { 103. Rhizoclonium riparium } \\
\text { (Roth) Harvey var. imple- } \\
\text { xum (Dillwyn) Rosenvinge }\end{array}$ & 1 & & Ve & L & $\mathrm{Se}$ & $\mathrm{R}$ & & $\begin{array}{c}\mathrm{Pm} \\
\mathrm{Ee}\end{array}$ \\
\hline $\begin{array}{l}\text { Siphonocladaceae } \\
\text { 104. Struveopsis robusta (Se- } \\
\text { tchell et Gardner) Rhyne } \\
\text { et H. Robinson }\end{array}$ & & VO & $\mathrm{Ve} V \mathrm{~V}$ & M & Se & $\mathrm{R}$ & & $\mathrm{N}$ \\
\hline $\begin{array}{l}\text { 105. Phyllodictyon anastomosans } \\
\text { (Harvey) Kraft et M. J. } \\
\text { Wynne }\end{array}$ & $P$ & & $\mathrm{Ve}$ & $M$ & Ex & $\mathrm{R}$ & & \\
\hline
\end{tabular}


Cuadro 1. Continuación.

\begin{tabular}{|c|c|c|c|c|c|c|c|c|}
\hline \multirow{2}{*}{ Especies } & \multicolumn{2}{|c|}{ Presencia } & \multirow{2}{*}{$\begin{array}{l}\text { Repro- } \\
\text { ducción }\end{array}$} & \multirow{2}{*}{$\begin{array}{l}\text { Nivel } \\
\text { de } \\
\text { marea }\end{array}$} & \multirow{2}{*}{$\begin{array}{l}\text { Exp. } \\
\text { al } \\
\text { oleaje }\end{array}$} & \multirow{2}{*}{$\begin{array}{l}\text { Sus- } \\
\text { trato }\end{array}$} & \multirow{2}{*}{$\begin{array}{c}\text { Epífita } \\
\text { de }\end{array}$} & \multirow[t]{2}{*}{ Obs } \\
\hline & $\begin{array}{l}\text { Punta } \\
\text { Arena }\end{array}$ & $\begin{array}{l}\text { Cabo } \\
\text { Pulmo }\end{array}$ & & & & & & \\
\hline $\begin{array}{l}\text { Valoniaceae } \\
\text { 106. Ernodesmis verticillata } \\
\text { (Kützing) Boergesen }\end{array}$ & & V & Ve & M & Ex & $\mathrm{R}$ & & $\mathrm{N}$ \\
\hline $\begin{array}{l}\text { Bryopsidaceae } \\
\text { 107. Bryopsis hypnoides } \\
\text { Lamouroux }\end{array}$ & PV & VO & $\begin{array}{l}\text { Ve } \mathrm{Ve} \\
\mathrm{Ve}\end{array}$ & L M & Ex Se & $\mathrm{R}$ & & $\mathrm{Pm}$ \\
\hline $\begin{array}{l}\text { 108. B. pennata Lamouroux var. } \\
\text { minor J. Agardh }\end{array}$ & & O & Ve & M & Se & $\mathrm{R}$ & & \\
\hline Caulerpaceae & & & & & & & & \\
\hline 109. Caulerpa peltata Lamouroux & $\mathrm{Ol}$ & PVO & $\begin{array}{l}\text { Ve Ve } \\
\text { Ve Ve }\end{array}$ & L M & Se & $\begin{array}{l}\mathrm{R} \\
\mathrm{A}\end{array}$ & & $\mathrm{Pm}$ \\
\hline $\begin{array}{l}\text { 110. C. racemosa var. turbinata } \\
\text { (J. Agardh) Eubank }\end{array}$ & $P$ & & Ve & M & D & A & & \\
\hline $\begin{array}{l}\text { 111. C. sertularioides (S. Gmelin) } \\
\text { M. Howe }\end{array}$ & $\mathrm{P}$ & PVO & $\begin{array}{l}\text { Ve Ve } \\
\text { Ve }\end{array}$ & L & $\mathrm{Se}$ & A & & $\mathrm{Pm}$ \\
\hline $\begin{array}{l}\text { Codiaceae } \\
\text { 112. Codium picturatum Pedro- } \\
\text { che et P. C. Silva }\end{array}$ & $\mathrm{PI}$ & $\mathrm{PO}$ & $\begin{array}{l}\text { Ve Gg } \\
\text { Gg }\end{array}$ & L M & Ex Se & $\mathrm{R}$ & & \\
\hline $\begin{array}{l}\text { Udoteaceae } \\
\text { 113. Chlorodesmis hildebrandtii } \\
\text { A. et E. S. Gepp }\end{array}$ & $P$ & & $\mathrm{Ve}$ & M & $\mathrm{D}$ & $\mathrm{R}$ & & \\
\hline $\begin{array}{l}\text { Dasycladaceae } \\
\text { 114. Neomeris annulata Dickie }\end{array}$ & $\mathrm{PI}$ & & Gg Gg & L & Ex & $\mathrm{R}$ & & $\mathrm{N}$ \\
\hline $\begin{array}{l}\text { Polyphysaceae } \\
\text { 115. Acetabularia parvula Solms } \\
\text { Laubach }\end{array}$ & $\mathrm{POI}$ & V & $\begin{array}{l}\text { Gg Gg } \\
\text { Ve Gg }\end{array}$ & M & D & $\mathrm{R}$ & & NCG \\
\hline
\end{tabular}

Presencia

$\mathrm{P}=$ Mayo, 1989; Abril, 1994

$\mathrm{V}=$ Julio, 1992

$\mathrm{O}=$ Octubre, 1989

I = Diciembre, 1992; Febrero, 1989

Reproducción

$\mathrm{Ht}=$ Heterocistos

$\mathrm{Hm}=$ Hormogonios

$\mathrm{Mn}=$ Monosporangios

$\mathrm{Te}=$ Tetrasporangios
$\mathrm{Bp}=$ Bisporangios

$\mathrm{Gf}=$ Gametos femeninos

$\mathrm{Ci}=$ Cistocarpos

$\mathrm{Gm}=$ Gametos masculinos

$\mathrm{Pp}=$ Esporangios pluriloculares

$\mathrm{Uu}=$ Esporangios uniloculares

$\mathrm{Gg}=$ Gameto vesiculoso

$\mathrm{Ps}=$ Polisporangios

$\mathrm{Ve}=$ Vegetativo 
Mateo-Cid et al.: Contribución al Estudio de las Algas Marinas Bentónicas

Nivel de marea

$\mathrm{L}=$ Intermareal

$M=$ Submareal

(Colecta por buceo libre entre 1-4

metros de profundidad)

Exposición al oleaje

$\mathrm{D}=$ Protegido

$\mathrm{Se}=$ Semiexpuesto

$\mathrm{Ex}=$ Expuesto

Sustrato

$\mathrm{R}=$ Rocoso

$A=$ Arenoso
Epífita de

Los números de esta columna corresponden a las algas sobre las que se localizaron las especies epífitas

\section{Observaciones}

NCG = Nueva cita para el Golfo de California

$\mathrm{N} \quad=$ Nueva cita para Cabo Pulmo

$\mathrm{Pm}=$ Pozas intermareales

PAR = Parásita

$\mathrm{Ee} \quad=$ Epífita estricta

\section{AGRADECIMIENTOS}

Se dan las gracias al Centro Interdisciplinario de Ciencias Marinas del Instituto Politécnico Nacional, que proporcionó el transporte para el traslado de personal y equipo. Y. E. Rodríguez-Montesinos, I. Sánchez y R. Robles colaboraron en la recolección del material biológico. Dante Espinosa Cid elaboró las figuras. Ma. Guadalupe Tejeda Hernández capturó la información.

\section{LITERATURA CITADA}

Abbott, I. A. y G. J. Hollenberg. 1976. Marine algae of California. Stanford University Press. Stanford, California. 827 pp.

Aguilar-Rosas, R. y M. A. Aguilar-Rosas. 1994. Estudio florístico de las algas marinas bentónicas del ejido San José, Baja California, México. Ciencias Marinas 20 (4): 511-534.

Anagnostidis, K. y J. Komárek. 1988. Modern approach to the classification system of Cyanophytes. 3. Oscillatoriales. Arch. Hydrobiol. Suppl. 80(1-4): 327-472.

Anaya, R.G. y R. Riosmena. 1996. Macroalgas del arrecife coralino de Cabo Pulmo-Los Frailes, B.C.S., México. Revista Biol. Trop. 44(2): 903-906.

Anómino. 1990. Calendario gráfico de mareas 1990. Universidad Nacional Autónoma de México. México, D.F. 72 pp.

Brusca, R. C. y D. B. Thompson. 1975. Pulmo Reef: the only "coral reef» in the Gulf of California. Ciencias Marinas 2: 37-53.

Cormaci, M. y G. Furnari. 1987. Nomenclatural notes on some Mediterranean algae. Taxon 36: 755758.

Dawes, C. J. 1986. Botánica Marina. Limusa. México, D.F. 673 pp.

Dawson, E. Y. 1944. Marine algae of the Gulf of California. Allan Hancock Pacific Expedition 3: 189454.

Dawson, E. Y. 1949. Resultados preliminares de un reconocimiento de las algas marinas de la costa pacífica de México. Rev. Soc. Mex. Hist. Nat. 9 (3-4): 215-254.

Dawson, E. Y. 1953a. Marine red algae of Pacific Mexico. I. Bangiales to Corallinaceae subfamily Corallinoideae. Allan Hancock Pacific Expeditions 17(1): 1-239. 
Dawson, E. Y. 1953b. Resumen de las investigaciones recientes sobre algas marinas de la costa pacífica de México con una sinopsis de la literatura, sinonimia y distribución de las especies descritas. Rev. Soc. Mex. Hist. Nat. 13: 97-197.

Dawson, E. Y. 1954. Marine red algae of Pacific Mexico. II. Cryptonemiales. Allan Hancock Pacific Expedition 17(2): 241-397.

Dawson, E. Y. 1958. Notes on Pacific coast marine algae. VII. Bull. Calif. Acad. Sci. 57: 65-80.

Dawson, E. Y. 1959. Marine algae of the 1958 cruise of Stella Polaris in the Gulf of California. Los Angeles County Museum Contributions to Science 27: 1-39.

Dawson, E. Y. 1960a. Marine red algae of Pacific Mexico. III. Cryptonemiales, Corallinaceae subfamily Melobesioideae. Pacific Naturalist 2(1): 3-125.

Dawson, E. Y. 1960b. The biogeography of Baja California and adyacent seas. II. Marine biotas, a review of the ecology, distribution and affinities of the benthic flora. Systematic Zoology 9: 93-100.

Dawson, E. Y. 1961. Marine red algae of Pacific Mexico. IV. Gigartinales. Pacific Naturalist 2(5): 191343.

Dawson, E. Y. 1962. Marine red algae of Pacific Mexico. VII. Ceramiales: Ceramiaceae, Delesseriaceae. Allan Hancock Pacific Expeditions 26(1): 1-207.

Dawson, E. Y. 1963a. Marine red algae of Pacific Mexico. VI. Rhodymeniales. Nova Hedwigia 5: 437476.

Dawson, E. Y. 1963b. Marine red algae of Pacific Mexico. VII. Ceramiales: Dasyaceae, Rhodomelaceae. Nova Hedwigia 6: 401-481.

Dawson, E. Y. 1966a. Marine botany: an introduction. Holt, Rinearth \& Winston. Nueva York. 371 pp.

Dawson, E. Y. 1966b. New records of marine algae from Gulf of California. J. Arizona Academy of Sciences 4: 55-66.

Dawson, E. Y. 1966c. Marine algae in the vicinity of Puerto Peñasco, Sonora, Mexico. University of Arizona Gulf of California Field Guide Series. University of Arizona. Tucson. No. 1. 157 pp.

Díaz-Piferrer, M. 1967. Las algas superiores y fanerógamas marinas. In: Ecología Marina. Fundación La Salle. Caracas. pp. 273-307.

García, E. 1981. Modificaciones al sistema de clasificación climática de Köppen (adaptada para la República Mexicana). Instituto de Geografía, Universidad Nacional Autónoma de México. México, D.F. 243 pp.

Hariot, P. 1895. Algues du Gulf de Californie recuellies par M. Diguet. J. Bot. 9: 167-170.

Hollenberg, G. J. 1961. Marine red algae of Pacific Mexico. V. The genus Polysiphonia. Pacific Naturalist 2(5-6):345-375.

Hollenberg, G. J. y J. N. Norris. 1977. The red algae Polysiphonia (Rhodomelaceae) in the northern Gulf of California. Smithsonian Contr. Marine Sciences 1: 1-21.

Howe, M. A. 1911. Phycological studies. V. Some marine algae of Lower California, Mexico. Bull. Torrey Bot. Club 38: 489-514.

Huerta-Múzquiz, L. y A. C. Mendoza-González. 1985. Algas marinas de la parte sur de Bahía de La Paz, Baja California Sur, México. Phytologia 59: 35-57.

Keats, D. W., Y. M. Chamberlain y M. Baba. 1997. Pneophyllum conicum (Dawson) comb. nov. (Rhodophyta, Corallinaceae), a widespread Indo-Pacific non-geniculate coralline alga that overgrows and kills live coral. Bot. Mar. 40: 263-279.

Komárek, J. y K. Anagnostidis. 1986. Modern approach to the clasification system of Cyanophytes 2. Chroococcales. Arch. Hydrobiol. Suppl. 73(2): 157-226.

Kornman, P. 1989. Sahlingia nov. gen. based on Erythrocladia subintegra (Erythropeltidales, Rhodophyta). Brit. Phycol. J. 24: 223-228.

Koster, J. T. 1955. The genus Rhizoclonium Kützing in the Netherlands. Pubblicazioni della Stazione Zoologica di Napoli 27: 335-357.

Kraft, G. T. y M. J. Wynne. 1996. Delineation of the genera Struvea Sonder and Phyllodictyon J. E. Gray (Cladophorales, Chlorophyta). Phycol. Research 44: 129-142. 
Littler, M. M., D. S. Littler y P. R. Taylor. 1983. Evolutionary strategies in a tropical barrier reef system: functional-form groups of marine macroalgae. J. Phycol. 19: 229-237.

Lüning, K. 1990. Seaweeds. Their enviroment, biogeography and ecophysiology. Wiley Interscience Publications. Nueva York. 527 pp.

Mateo-Cid, L. E., I. Sánchez-Rodríguez, Y. E. Rodríguez-Montesinos y M. M. Casas-Valdez. 1993. Estudio florístico de las algas marinas bentónicas de Bahía Concepción, B. C. S., México. Ciencias Marinas 19(1): 41-60.

Mendoza-González, A. C. y L. E. Mateo-Cid. 1986. Flora marina bentónica de las costa noroeste del estado de Sonora, México. Phytologia 60(6): 414-427.

Norris, J. N. 1972. Marine algae of the 1969 cruise of Markele to the northern part of the Gulf of California. Bol. Soc. Bot. México. 32: 1-30.

Norris, J. N. y H. W. Johansen. 1981. Articulated coralline algae of the Gulf of California, México. I: Amphiroa Lamouroux. Smithsonian Contribution to the Marine Sciences 9: 1-29.

Norris, R. E. y J. N. Norris. 1973. Kallymenia pertusa (Rhodophyceae, Crytonemiales) from the Gulf of California. Phycologia 12: 71-74.

Osorio-Taffall, B. F. 1943. El Mar de Cortés y su productividad fitoplanctónica. Anales Esc. Nac. Ci. Biol. 3(1-2): 73-118.

Pacheco-Ruiz, I., J. A. Zertuche-González, P. Cabello-Pasini y B. H. Brinkhuis. 1992. Growth responses and seasonal biomass variation of Gigartina pectinata Dawson (Rhodophyta) in the Gulf of California. J. Exp. Mar. Biol. Ecol. 157: 263-274.

Pedroche, F. F. Y P. C. Silva. 1996. Codium picturatum sp. nov. (Chlorophyta), una especie extraordinaria del Pacífico tropical mexicano. Acta Bot. Mex. 35: 1-8.

Rocha-Ramírez, V. y D. A. Siqueiros-Beltrones. 1991. El herbario ficológico de la U. A. B. C. S.: Elenco florístico de las macroalgas para Balandra en la Bahía de la Paz, B. C. S. México. Rev. Inv. Cient. Universidad Autónoma de Baja California Sur 2: 13-34.

Round, F. E. 1981. The ecology of the algae. Cambridge University Press. Nueva York. 653 pp.

Santelices, B. 1977. Ecología de algas marinas bentónicas. Documento de la Dirección General de Investigaciones, Univ. Católica de Chile. Santiago de Chile. 488 pp.

Setchell, W. A. y N. L. Gardner. 1924. New marine algae of the Gulf of California. Proc. Calif. Acad. Sci. 12 (4): 695-949.

Silva, P. C. 1991. Notes on the type specimens of red algae parasites described from California by W. A. Setchell. Taxon 40: 463-470.

Silva, P. C., E. G. Meñez y R. L. Moe. 1987. Catalogue of the benthic marine algae of the Philippines. Smithsonian Contr. Marine Sciences 27: 1-179.

Silva, P. C., P. W. Basson y R. L. Moe. 1996. Catalogue of the benthic marine algae of the Indian Ocean. Univ. Calif. Publ. Bot. 79. 1259 pp.

Taylor, W. R. 1945. Pacific marine algae of the Allan Hancock Pacific Expeditions to the Galapagos Islands. Allan Hancock Pacific Expeditions 12: 1-528.

Taylor, W. R. 1960. Marine algae of the eastern tropical and subtropical coasts of the Americas. University of Michigan Press. Ann Arbor. 870 pp.

Werner, D. (ed.) 1977. The biology of diatoms. Blackwell Scientific Publications. Oxford. 478 pp.

Wynne, M. J. 1986. A checklist of benthic marine algae of the tropical and subtropical western Atlantic. Can. J. Bot. 64: 2239-2281. 